\title{
Influence of Vitroperm Content on the Energy Losses in Composite Materials Based on the Mixture of Two Ferromagnets
}

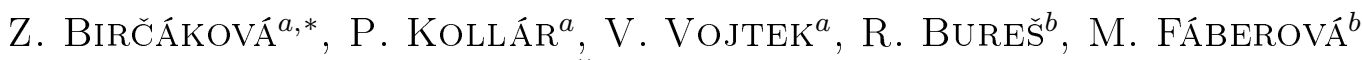 \\ ${ }^{a}$ Institute of Physics, Faculty of Sciences, P. J. Šafárik University, Park Angelinum 9, 04154 Košice, Slovakia \\ ${ }^{b}$ Institute of Materials Research SAS, 04001 Košice, Watsonova 47, 04001 Košice, Slovakia
}

\begin{abstract}
Soft magnetic composites offer several advantages such as 3D isotropic magnetic properties and relatively small energy losses, finding use in electrical devices like electromotors, transformers or sensors. The aim of this work was to investigate the dc and ac magnetic properties of composites based on the mixture of two different ferromagnets: iron based material Somaloy ${ }^{\circledR} 700$ and Vitroperm ${ }^{\circledR} 500$ alloy. The analysis of total losses into dc losses, classical losses and excess losses showed that the classical and excess losses were negligibly small. The specific resistivity was increasing with the increasing fraction of Vitroperm (VPM). The coercivity exhibited maximum at $20 \%$ of VPM.
\end{abstract}

DOI: $10.12693 /$ APhysPolA.126.114

PACS: 75.50.Bb, 75.60.Jk, 75.60.-d

\section{Introduction}

Soft magnetic composites (SMCs) have been used in various applications due to their special properties like low core losses, isotropic magnetic properties and high electrical resistivity. SMCs consist of small ferromagnetic powder particles surrounded by an insulating film $[1,2]$.

The aim of this study was to investigate the contributions of the dc losses, the classical and the excess losses to the total losses in the frequency range from dc to $100 \mathrm{~Hz}$ (where the contributions of losses are best observable) of composite materials based on the mixture of two different ferromagnets.

\section{Results and discussion}

In this work we investigated samples consisting of mixture of materials: Somaloy ${ }^{\circledR} 700$ [3] (polycrystalline iron powder covered by insulating film) provided by Höganäs AB, Sweden, and Vitroperm ${ }^{\circledR} 500$ [4] (VPM) alloy in the form of flakes (originally amorphous transformed into partialy nanocrystalline after annealing) provided by Vacuumschmelze, GmbH \& Co. KG, Germany. The SMC samples were prepared by conventional powder metallurgy in the form of a ring (outer diameter of about $24 \mathrm{~mm}$, inner diameter of $18 \mathrm{~mm}$, height about $1.5 \mathrm{~mm}$ ) and a cylinder (outer diameter of about $10 \mathrm{~mm}$, height about $1.5 \mathrm{~mm}$ ) by mixing Somaloy powder with weight fractions of $5,10,20,30$ and 50 wt.\% of VPM powder. Mixed powder was compacted in a cylindrical die at uniaxial pressure of $800 \mathrm{MPa}$ and then cured at temperature of $530{ }^{\circ} \mathrm{C}$ for $60 \mathrm{~min}$. in an electric furnace in air. Parameters of the samples are in Tab. I.

The morphology of samples was documented using SEM (TESLA BS 340). The photographs of materials

\footnotetext{
*corresponding author; e-mail: zuzka.bircakova@szm.sk
}

before pressing and also the structure of final composites are shown in Ref. 5. The dc and ac hysteresis loops (up to $100 \mathrm{~Hz}$ ) were measured by fluxmeter-based hysteresisgraphs, only a fraction of magnetic material was taken in account. The coercivity was measured by Foerster Koerzimat. The four contact method was used for the specific electrical resistivity measurements.

Parameters of the samples.

TABLE I

\begin{tabular}{|c|c|c|c|c|c|}
\hline Sample & $\begin{array}{c}\text { S95 } \\
5 \mathrm{VPM}\end{array}$ & $\begin{array}{c}\text { S90 } \\
10 \mathrm{VPM}\end{array}$ & $\begin{array}{c}\mathrm{S} 80 \\
20 \mathrm{VPM}\end{array}$ & $\begin{array}{c}\text { S70 } \\
30 \mathrm{VPM}\end{array}$ & $\begin{array}{c}\text { S50 } \\
50 \mathrm{VPM}\end{array}$ \\
\hline $\begin{array}{l}\text { Somaloy to } \\
\text { VPM ratio }\end{array}$ & $95: 5$ & $90: 10$ & $80: 20$ & $70: 30$ & $50: 50$ \\
\hline Porosity (\%) & 1 & 4 & 10 & 13 & 18 \\
\hline Density $\left(\mathrm{g} / \mathrm{cm}^{3}\right)$ & 7.61 & 7.33 & 6.86 & 6.62 & 6.12 \\
\hline$W_{d c}\left(\mathrm{~J} / \mathrm{m}^{3}\right)^{*}$ & 57.3 & 65.7 & 75.0 & 76.6 & 68.8 \\
\hline$W_{c}^{\text {inter }}\left(\mathrm{J} / \mathrm{m}^{3}\right)^{*}$ & 0.057 & 0.071 & 0.080 & 0.062 & 0.020 \\
\hline$W_{c}^{\text {intra }}\left(\mathrm{J} / \mathrm{m}^{3}\right)^{*}$ & 0.28 & 0.26 & 0.24 & 0.21 & 0.15 \\
\hline$W_{e x c}\left(\mathrm{~J} / \mathrm{m}^{3}\right)^{*}$ & 7.07 & 7.17 & 14.38 & 13.13 & 13.03 \\
\hline
\end{tabular}

The total energy losses $W_{\text {tot }}$ (in $\mathrm{J} / \mathrm{m}^{3}$ ) dissipated in magnetic material are the sum of three components: $\mathrm{dc}$ losses $W_{d c}$, classical losses $W_{c}$ and excess losses $W_{\text {exc }}[6]$. The dc losses $W_{d c}$ are represented as the area of dc hysteresis loop, the classical losses $W_{c}$ are caused by the eddy currents induced by changing magnetic flux, and the excess losses $W_{\text {exc }}$ are related mainly to number of moving domain walls during the magnetization process $[7,8]$. We distinguish intra-particle (for insulated particles) and inter-particle (for non-perfect insulated particles) eddy currents. The inter-particle classical losses $W_{c}^{\text {inter }}$ can be expressed $[6,9]$ :

$$
W_{c}^{\text {inter }}=\frac{\left(\pi d_{e f f} B_{m}\right)^{2}}{\beta \rho_{R}^{\text {bulk }}} f,
$$

where $d_{e f f}$ is effective dimension for eddy current, $B_{m}$ is maximum flux density, $f$ frequency, $\rho_{R}^{b u l k}$ specific resistivity and $\beta$ is geometrical coefficient, which for rectangular cross-section is $\beta=6 /(1-0.633(b / a) \tanh (1.58 a / b))$, 
$a$ and $b$ are the lengths of sides of the rectangle [9], $b \leq a$. For the calculation of intra-particle classical losses $W_{c}^{\text {intra }}$ Eq. 1 can also be used with the specific resistivity of magnetic material $\rho_{R}$ instead of $\rho_{R}^{b u l k}$ and for spherical magnetic particles $(\beta=20[2])$ the particle diameter as $d_{\text {eff }}$.

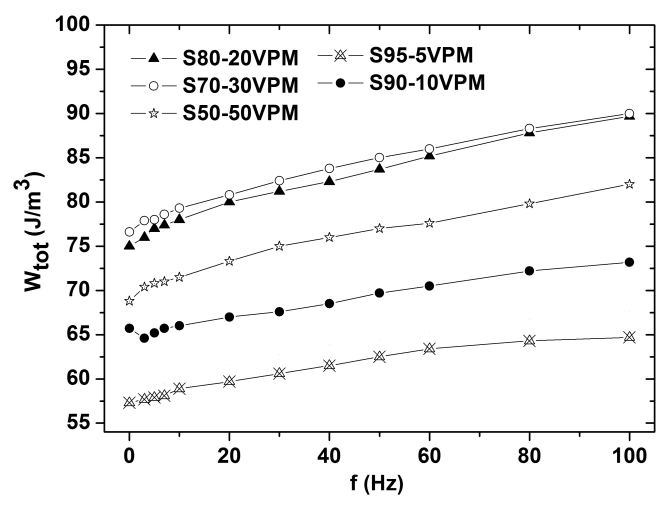

Fig. 1. Total energy losses as a function of frequency at $B_{m}=0.2 \mathrm{~T}$.

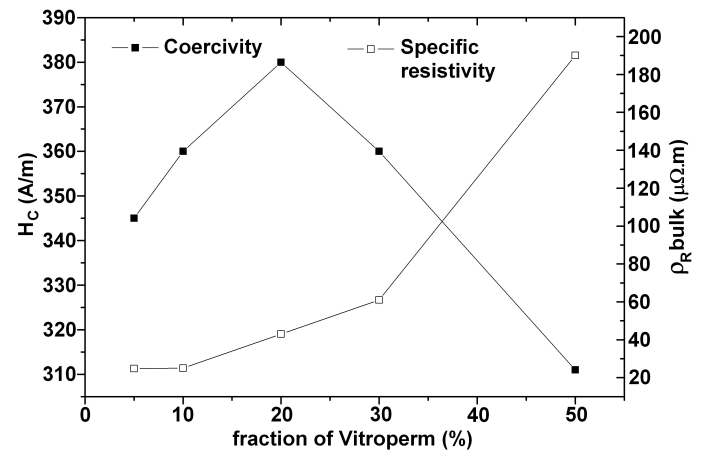

Fig. 2. The coercivity and specific resistivity vs. VPM fraction.

The dependencies of total losses versus frequency $(\mathrm{dc}-100 \mathrm{~Hz})$ at maximum flux density of $0.2 \mathrm{~T}$ are depicted in Fig. 1. The dependencies are not linear, which means that numbers of moving domain walls are not constant [7]. We see that with the increasing VPM content the energy losses increase, but the sample with the highest VPM fraction (50\%) exhibits lower losses than samples with 20 and $30 \%$ (in spite of higher porosity Tab. I). In Fig. 2 the coercivity $H_{c}$ and the specific resistivity $\rho_{R}^{\text {bulk }}$ as functions of VPM content are shown. $H_{c}$ exhibits maximum at $20 \%$ of VPM and $\rho_{R}^{\text {bulk }}$ increases with the increasing VPM content, which is useful for lowering the inter-particle classical losses.

Total energy losses were separated into four components (Tab. I). The inter-particle and intra-particle classical losses were calculated from Eq. 1, where required values of $\rho_{R}^{\text {bulk }}$ are mentioned in Tab. I, $\rho_{R}$ is specific resistivity of iron in Somaloy (tabular value: $0.098 \mu \Omega \cdot \mathrm{m}$ [3]) and specific resistivity of nanocrystalline VPM in the form of ribbon (tabular value: $1.2 \mu \Omega \cdot \mathrm{m}$ [4]). Particle diameters were obtained by SEM, whereby observed value for spherical particles of Somaloy is $120 \mu \mathrm{m}$. Flat ir- regular shaped particles of VPM with random orientation and dimensions $200 \times 200 \times 20 \mu \mathrm{m}$ were abstracted by cubic form with edge $90 \mu \mathrm{m}$ long. The excess losses $W_{\text {exc }}$ are the difference between the total losses and the sum of classical and dc losses. We see that dc losses are the majority component in comparison with other components (together representing the dynamic losses), from which $W_{\text {exc }}$ are dominant. That is explained by the quite low numbers of moving domain walls [8]. Both the components of classical losses are negligibly small and are furthermore decreasing with increasing VPM fraction, which is valuable especially at higher frequencies.

\section{Conclusion}

In this work the dc and ac magnetic properties of SMCs based on the mixture of Somaloy ${ }^{\circledR} 700$ and Vitroperm ${ }^{\circledR}$ 500 (VPM) were investigated. Total energy losses were analysed into components (dc losses, classical losses and excess losses) and compared in relation to VPM to Somaloy ratio.

We conclude that with the increasing VPM content the specific resistivity was increasing and the coercivity showed maximum at $20 \%$ of VPM. In sample with $50 \%$ of VPM the total energy losses were lower than in samples with 20 and $30 \%$ of VPM. Classical losses were negligibly small and were decreasing with the increasing VPM fraction.

\section{Acknowledgments}

This work was supported by projects nanoCEXmat I, ITMS 26220120019 and nanoCEXmat II, ITMS: 26220120035, of Operational Program "Research and Development" financed through European Regional Development Fund; by Slovak Research and Development Agency under contract APVV-0222-10 MAGCOMP; by Scientific Grant Agency of Ministry of Education of Slovak Republic and Slovak Academy of Sciences, VEGA 1/0861/12 and by P. J. Šafárik University grants: VVGSPF-2013-106 and VVGS-2013-107. Special thanks to Höganäs AB Sweden for providing Somaloy ${ }^{\circledR}$ powder and to Mr. M. Vitovský, Vacuumschmelze, GmbH \& Co. KG, Germany for providing Vitroperm ${ }^{\circledR} 500$.

\section{References}

[1] H. Shokrollahi, K. Janghorban, J. Mater. Proc. Technol. 189, 1 (2007).

[2] A. Taghvaei, H. Shokrollahi, K. Janghorban, H. Abiri, Mater. Design 30, 3989 (2009).

[3] http://www.hoganas.se.

[4] http://ww. vacuums chmelze.de.

[5] J. Füzerová, J. Füzer, P. Kollár, L. Hegedüs, R. Bureš, M. Fáberová, IEEE Trans. on Mag. 48, 1545 (2012).

[6] B.D. Cullity, C.D. Graham, Introduction to magnetic materials, Wiley IEEE Press, Piscataway, NJ 2009.

[7] P. Kollár, Z. Birčáková, J. Füzer, R. Bureš, M. Fáberová, J. Magn. and Magn. Mater. 327, 146 (2013).

[8] M. Anhalt, J. Magn. Magn. Mater. 320, e366 (2008).

[9] T. D. Shen, U. Harms, R. B. Schwarz, Mater. Sci. Forum 386, 441 (2002). 